reflect the therapeutic action of VPA in AD, and they emphasize that this could have general implications for monitoring treatment effects in neurodegenerative disease.

Original article Mhyre TR et al. (2007) Proteomic analysis of peripheral leukocytes in Alzheimer's disease patients treated with divalproex sodium. Neurobiol Aging [doi:10.1016/j.neurobiolaging.2007.04.004]

\section{Alternating electric fields disrupt glioblastoma tumor growth in pilot study}

Low-intensity, intermediate-frequency electric fields of alternating direction ('tumor-treating fields'; TTFields) have been shown to halt cancer growth in culture and in mouse dermal tumors by disrupting cytokinesis. Now, Kirson et al. have extended their earlier experiments to show that TTFields effectively and safely slow tumor progression in various animal models of cancer and in humans with recurrent glioblastoma multiforme, a highly treatment-resistant brain tumor.

In the animal study, multidirectional TTFields were applied via external electrodes to tumors in rats inoculated with glioma cells. The growth of treated tumors was significantly inhibited compared with control tumors, and no treatmentrelated toxicity was noted. On the basis of these promising results, the investigators initiated a single-arm pilot study of TTFields treatment in 10 patients with glioblastoma multiforme. The median overall survival of TTFields-treated patients was more than double that of a literature-based historical control group (62.2 weeks versus $29.3 \pm 6$ weeks), and the rate of progression-free survival at 6 months was $50 \%(95 \% \mathrm{Cl} 23-77 \%)$ in TTFields-treated patients, compared with $15.3 \pm 3.8 \%$ in controls. Furthermore, the treatment was extremely well tolerated and virtually no adverse effects were observed.

This small pilot study represents the first report of the safety and efficacy of TTFields for treatment of cancer. Although the results are preliminary, TTFields could represent a new method for arresting proliferation and inducing cell death in tumors. A pivotal multicenter clinical trial is currently in progress in the US and Europe.

Original article Kirson ED et al. (2007) Alternating electric fields arrest cell proliferation in animal tumor models and human brain tumors. Proc Natl Acad Sci USA 104: 10152-10157

\section{Exposure to haloperidol after methamphetamine causes excitotoxicity}

The antipsychotic drug haloperidol is often used in emergency care to treat psychoses induced by methamphetamine, but a recently published animal study by Hatzipetros et al. warns that this therapy might cause neuronal death in the substantia nigra (SN) and could predispose individuals to hyperkinetic movement disorders and seizures.

A novel toxicity to $\gamma$-aminobutyric acid (GABA)releasing cells was observed in the $\mathrm{SN}$ pars reticulata ( $\mathrm{SNr}$ ) of adult rats treated with subchronic haloperidol after administration of high doses of methamphetamine. The loss of GABA neurons in this treatment group was demonstrated by significant decreases in the expression of neuronal-specific nuclear protein $(P<0.05)$ and glutamate decarboxylase $67(P<0.05)$ compared with controls; death of GABAergic cells in the SNr of treated rats was confirmed by the presence of increased DNA fragmentation. Dopaminergic neurons in the SN were unaffected. Further experiments demonstrated that neither methamphetamine nor haloperidol administration alone caused loss of GABAergic neurons, but that loss of these cells did result from exposure to both drugs combined. The investigations showed that $D_{2}$ receptor antagonism (with haloperidol) during or after methamphetamine administration causes a persistent release of glutamate in the SNr, activation of glutamate receptors, and subsequent excitotoxicity.

Hatzipetros et al. conclude that the death of GABAergic cells in the SN contraindicates the treatment of methamphetamine-induced psychoses with haloperidol and that current clinical practices may need to be reconsidered.

\section{Original article Hatzipetros T et al. (2007) Haloperidol treatment after high-dose methamphetamine administration is excitotoxic to GABA cells in the substantia nigra pars reticulata. J Neurosci 27: 5895-5902 \\ Human herpesvirus-6B implicated in the etiology of mesial temporal lobe epilepsy}

Mesial temporal lobe epilepsy (MTLE) is a common and intractable form of seizure disorder that is characterized by extensive hippocampal 